\title{
Extensively drug-resistant tuberculosis (XDR-TB) among health care workers in South Africa
}

\author{
Julie Jarand ${ }^{1,2}$, Karen Shean ${ }^{2}$, Max O’Donnell ${ }^{3}$, Marian Loveday ${ }^{4}$, Charlotte Kvasnovsky ${ }^{5,6}$, Martie Van der Walt ${ }^{6}$, \\ Shahieda Adams', Paul Willcox ${ }^{2}$, Justin O'Grady ${ }^{7}$, Alimuddin Zumla ${ }^{7}$ and Keertan Dheda ${ }^{1,7}$ \\ 1 Lung Infection and Immunity Unit, Department of Medicine, University of Cape Town, South Africa \\ 2 University of Calgary, Calgary, Canada \\ 3 Boston University School of Medicine, Boston, MA, USA \\ 4 South African Medical Research Council, Tygerberg, South Africa \\ 5 Emory University School of Medicine \& Rollins School of Public Health, Atlanta, GA, USA \\ 6 South African Medical Research Council, Pretoria, South Africa \\ 7 Centre for Infectious Diseases and International Health, University College London Medical School, UK
}

Summary OвJестіve To determine the clinical profile and outcomes of health care workers (HCWs) with extensively drug resistant tuberculosis (XDR-TB) in the Eastern and Western Cape Provinces of South Africa.

METHOD Retrospective case record review of 334 patients with XDR-TB reported during the period 1996-2008 from Western and Eastern Cape Province, Cape Town, South Africa. Case records of HCWs with XDR-TB were analysed for clinical and microbiological features, and treatment outcomes.

RESULTS From 334 case records of patients with XDR-TB, 10 HCWs were identified. Eight of ten were HIV-uninfected, and four of 10 had died of XDR-TB despite treatment. All $10 \mathrm{HCWs}$ had received an average of 2.4 courses of TB treatment before being diagnosed as XDR-TB.

CONCLUSIONS In the Eastern and Western Cape provinces of South Africa XDR-TB affects HCWs, is diagnosed rather late, does not appear to be related to HIV status and carries a high mortality. There is an urgent need for the South African government to implement WHO infection control recommendations and make available rapid drug susceptibility testing for HCWs with suspected multidrug-resistant (MDR)/XDR-TB. Further studies to establish the actual risk and sources of infection (nosocomial or community) are required.

keywords extensively drug-resistant, tuberculosis, health worker

\section{Introduction}

Multidrug (MDR)- and extensively drug (XDR)-resistant tuberculosis $(\mathrm{TB})$ carry a high mortality rate and are a growing problem that threatens to destabilize tuberculosis control globally (WHO Report. World Health Organization, 2010; WHO, 2010; Centers for Disease Control and Prevention, 2006; Sotgiu et al. 2009; Gandhi et al. 2006). South Africa has a very high annual TB incidence of 948 per 100000 population/year with approximately 80009000 patients per annum with multidrug-resistant TB (MDR-TB) passively detected per annum. The most recent report of $5 \%$ of the patients with MDR-TB being extensively drug-resistant TB (XDR-TB) in South Africa is likely an underestimate (WHO Report. World Health Organization, 2010; WHO, 2010). TB in all its forms is having a devastating effect on the world's population and poses a great risk to the health and work outputs of health care workers (HCWs) (Menzies et al. 2007; Naidoo \& Jinabhai 2006; Jo et al. 2008; Pillay \& Malhati 2008; Galgalo et al. 2008; Joshi et al. 2006).

Health care workers in South Africa are at the forefront of the battle against the TB and HIV/AIDS epidemics (Naidoo \& Jinabhai 2006; Joshi et al. 2006; Shisana et al. 2004). The shortage of HCWs has reached alarming levels in sub-Saharan Africa, which is home to $11 \%$ of the world's population and $24 \%$ of the global burden of disease, but only $3 \%$ of the world's HCWs (Wilkinson \& Gilks 1998). Multiple factors contribute to this problem, such as high workload, poor remuneration, limited training opportunities and lack of resources (Pillay \& Malhati 2008). Other significant contributing factors are fatalities and incapacitating disability related to HIV/AIDS and other infectious diseases of poverty. In 2002, an estimated 
J. Jarand et al. XDR-TB among health care workers in South Africa

$16 \%$ of HCWs in South Africa were infected with HIV (Shisana et al. 2004). South Africa has one of the highest rates of TB and HIV/AIDS in the world, and the occurrence of TB in HCWs has been reported to be substantial (Naidoo \& Jinabhai 2006; Shisana et al. 2004; Wilkinson \& Gilks 1998). However, the occurrence and importance of XDR-TB in HCWs in South Africa are not yet documented.

Health care workers with active tuberculosis (TB) need to be identified and treated. HCWs with active TB risk infecting their patients, their fellow staff, families and communities with Mycobacterium tuberculosis (Mtb). Conversely, HCWs are also at risk of acquiring TB from their patients, fellow staff and family and community members. Over the past 15 years, the risk of TB in HCWs in southern Africa has been increased because of the resurgence of TB caused by the HIV epidemic and the emergence of MDR-/XDR-TB, which has added significant strain to already overwhelmed TB control programmes (Gandhi et al. 2006; Naidoo \& Jinabhai 2006; Shisana et al. 2004). The prevalence of extensively drug-resistant tuberculosis (XDR-TB) is now being increasingly reported from South Africa (Gandhi et al. 2006; Dheda et al. 2010) and poses a major threat to HCWs and other patients in the wards and outpatient clinics, and those in congregate settings. XDR-TB is difficult to treat and has poor treatment outcomes, and together with HIV coinfection, may exacerbate the shortage and poor performance of HCWs in South Africa. The frequency, clinical profile and outcomes of HCWs with XDR-TB in South Africa remain unknown (Sotgiu et al. 2009). To ascertain whether XDR-TB is an emerging problem in HCWs in South Africa, we undertook a retrospective case record review of 334 patients with XDR-TB reported during the period 1996-2008 from the Eastern and Western Cape Provinces of South Africa.

\section{Methods and patients}

Using a validated data capture tool, we performed a retrospective study of case records of 334 patients with XDR-TB, diagnosed between January 1996 and February 2008, from the Western Cape and Eastern Cape provinces of South Africa. For the HCWs identified with XDR-TB, patient data, including demographics, microbiologic and treatment records, were collected. The diagnosis of XDRTB was based on sputum culture positivity for $M t b$ bacilli, and was defined, based on drug susceptibility testing (DST), as resistant to isoniazid, rifampicin, any one fluoroquinolone and at least one-second-line injectable anti-TB drug. Case definitions for diagnosis and conversion are outlined in Table 1.

\section{Results}

Ten HCWs with XDR-TB were identified. Table 1 outlines patient characteristics, including gender, HIV status, occupation, place of work, number of previous TB episodes, chest X-ray abnormalities, number of resistant drugs, treatment accorded and treatment outcomes. Patients were predominantly female (9 of 10) with a median age of 40 years (range 26-50). The majority of patients (6 of 10) worked as nursing staff and one each in the following professions: doctor, radiographer, laundry staff and ward cleaner. Of the 10 patients, eight were HIVuninfected. One HIV-positive patient had been treated with anti-retrovirals (ARVs) for 4 years prior to diagnosis of XDR-TB, and the other HIV-uninfected patient was started on ARVs at the time of XDR-TB diagnosis. All 10 patients had been treated for TB previously with an average of 2.4 previous TB episodes. The median duration of follow-up was 10 months (range 1-22 months) from the time of XDR-TB diagnosis.

Table 2 shows the individual patient characteristics (age, gender, year of diagnosis, occupation, HIV status, drugresistant patterns and treatment accorded and treatment outcome) of the $10 \mathrm{HCW}$ s with XDR-TB that were studied. Three patients underwent surgical resection because of failure to improve clinically, and they had unilateral disease. A high mortality was observed (4 of 10 patients died). Para-amino-salicylic acid (PAS) and capreomycin became available again in South African government-run hospitals in late 2006. Seven patients were treated with regimens containing these drugs, two patients were diagnosed and died before these drugs were available and one patient responded to a modified MDR-TB regimen which did not include capreomycin or PAS. Four patients died and six were still being maintained on treatment for XDR-TB at the end of the study.

\section{Discussion}

This case series study adequately answers our primary study aim, confirming that XDR-TB in HCWs is an important clinical problem, and should be of major concern to TB programme managers in South Africa. It affects a spectrum of HCWs, particularly nurses in the Western and Eastern Cape Provinces of South Africa. Whilst in MDR-TB sputum culture conversion is usually achieved in most patients within 3-4 months (WHO, 2010) of initiation of MDR-TB therapy, six of the 10 HCWs with XDR-TB in this study did not achieve culture conversion within this time frame. Moreover, two of the four converters reverted back to culture positivity prior to the end of follow-up, and the overall mortality was high 
J. Jarand et al. XDR-TB among health care workers in South Africa

Table I Summary characteristics of health care workers with XDR-TB

\begin{tabular}{|c|c|}
\hline & $N=10(\%)$ \\
\hline \multicolumn{2}{|l|}{ Gender } \\
\hline Male & 1 \\
\hline Female & 9 \\
\hline Median age in years (range) & $41(26-50)$ \\
\hline \multicolumn{2}{|l|}{ Year of XDR diagnosis* } \\
\hline 1996 & $1 \dagger$ \\
\hline 2002 & $1 \dagger$ \\
\hline 2006 & 2 \\
\hline 2007 & 4 \\
\hline 2008 & 2 \\
\hline \multicolumn{2}{|l|}{ HIV } \\
\hline Positive & 2 \\
\hline Negative & 8 \\
\hline Previous TB & $10(100 \%)$ \\
\hline Mean no. of previous TB episodes & 2.4 \\
\hline Median duration of follow-up (months) & 10 (range $1-22$ ) \\
\hline Surgical resection performed & $3(30 \%)$ \\
\hline \multicolumn{2}{|l|}{ Chest X-ray abnormalities } \\
\hline Bilateral, non-cavitary & 2 \\
\hline Bilateral, cavitary & 4 \\
\hline Unilateral, noncavitary & 1 \\
\hline Unilateral, cavitary & 2 \\
\hline Not recorded & 1 \\
\hline \multicolumn{2}{|l|}{ Occupation } \\
\hline Nurse & 5 \\
\hline Nursing student (3rd year) & 1 \\
\hline Physician & 1 \\
\hline Cleaner & 1 \\
\hline Laundry staff & 1 \\
\hline Radiographer & 1 \\
\hline \multicolumn{2}{|l|}{ Facilities where patient worked } \\
\hline District hospital & 3 \\
\hline Specialised TB hospital & 1 \\
\hline Prison & 1 \\
\hline Variable (nursing student) & 1 \\
\hline Physiotherapist office & 1 \\
\hline Not recorded & 3 \\
\hline Mean no. of resistant drugs & 5 \\
\hline $\begin{array}{l}\text { Median Time from MDR to XDR } \\
\text { (months) }\end{array}$ & 19 (range 0-74) \\
\hline $\begin{array}{l}\text { No. of pts who converted cultures } \\
\text { to negative }\end{array}$ & 4 \\
\hline $\begin{array}{l}\text { Median time to culture conversion } \\
\text { (months) } \mathbb{S}\end{array}$ & 1.7 (range 1.2-6.9) \\
\hline Mean no. of drugs in treatment regimen & 7 \\
\hline Outcomes & 7 \\
\hline Died & 4 \\
\hline Still on treatment & 6 \\
\hline
\end{tabular}

XDR-TB, extensively drug-resistant tuberculosis; MDR, multidrugresistant.

"Date of diagnosis is the date the first sputum, culture positive for XDR-TB.

$\dagger$ Patients were diagnosed with XD-RTB retrospectively with the 2006 definition of XDR-TB ${ }^{7}$

$\$$ Culture conversion is defined by having at least two consecutive negative sputum cultures, at least 1 month apart, and culture positivity at treatment initiation.

\$Time to culture conversion is duration from treatment start date to date of first negative culture.
$(40 \%)$. Thus, treatment-related outcomes seen in this limited case series of 10 patients were poor. This is concordant with other observations on treatment success (cured and completed) ranging from $34 \%$ to $67 \%$ and mortality of $7-36 \%$ in non-HCW populations (Sotgiu et al. 2009).

Health care workers acquiring XDR-TB adds to the growing burden of drug-resistant disease. It also concurrently exacerbates the shortage of HCWs as they can no longer work while undergoing prolonged inpatient treatment and during convalescence (Pillay \& Malhati 2008; Wilkinson \& Gilks 1998; Dheda et al. 2010; World Health Organization, 2006). This emphasises the need for intensified HCW screening, the widespread use of newer tools now available for the rapid diagnosis of MDR-/XDR-TB and instituting appropriate effective treatment. Importantly, the delay in diagnosis of XDR-TB in these HCWs indicates that undiagnosed and untreated, they are a risk to inpatients they manage, other fellow staff, and their families and communities.

Because of the limitations of our study design and the data obtained, it is unclear where and from whom the HCWs acquired the infection: in hospital as a nosocomial infection from inpatients with XDR-TB they cared for or from the community where XDR-TB is becoming more prevalent. Nosocomial transmission of drug-resistant $\mathrm{M} t b$ strains has been well documented and therefore HCWs are clearly at risk (Gandhi et al. 2006; Menzies et al. 2007). Given that HCWs in our study were previously treated for $\mathrm{TB}$, (including on at least two previous TB episodes in eight patients), community acquired, rather than nosocomial, XDR-TB may be a possibility. However, given the high prevalence of previous TB in patients with drug resistance in the Western Cape (Dheda et al. 2010) and studies demonstrating re-infection as an important modality of transmission (Verver et al. 2005), it is imperative that the transmission dynamics of these infections be ascertained. However, without identification of the $M t b$ strains and their molecular typing data from the HCWs studied, and from the index case, and from prior TB episodes, it was not possible to determine the transmission dynamics in the cases that we describe. The transmission patterns of drugresistant $M t b$ strains and whether XDR-TB may harbor different $M t b$ isolates and strains are unknown. Recent observations that $M t b$ strains have a rapid rate of molecular evolution at the hypervariable and immunogenic $M t b$ PPE38 gene region, which may lead to evasion by the immune system, may be important. It may also be that $M t b$ strains may be more diverse than previously thought (McEvoy et al. 2009). Prospective studies are now required to address these important issues for proper interventions to be developed. 
J. Jarand et al. XDR-TB among health care workers in South Africa

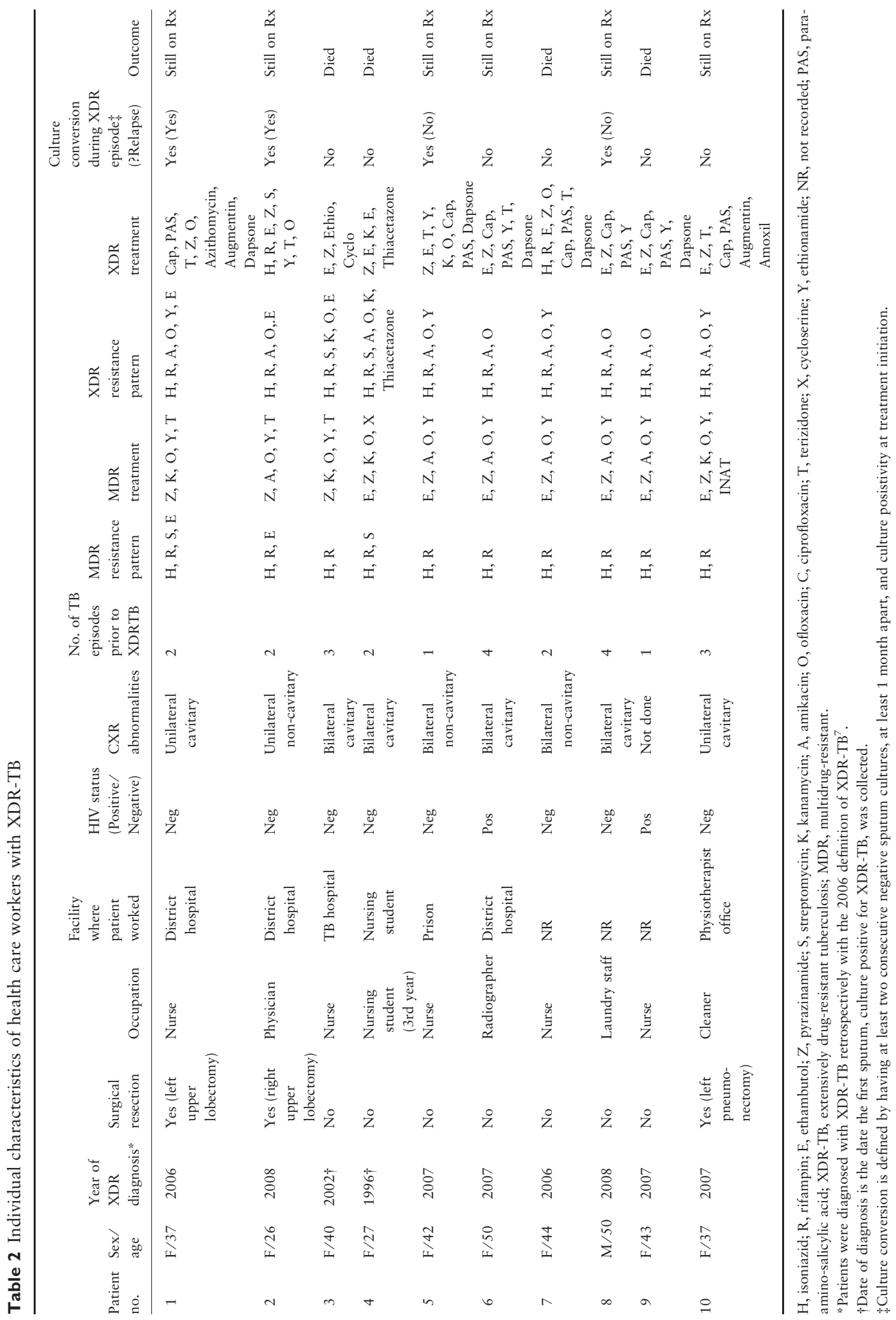


Although XDR-TB in South Africa was initially thought to be almost exclusively associated with HIV coinfection (Gandhi et al. 2006), this study shows that HIV-uninfected HCWs are also at risk of acquiring XDRTB. Therefore, MDR-/XDR-TB should be considered in any HCW suspected of TB, irrespective of HIV status, and all HCW should be screened with the newer rapid first- and second-line drug susceptibility tests if they are found to be smear or culture positive (Pai et al. 2006). All HCWs in South Africa should also be encouraged to be tested for HIV, and if HIV-infected, they should receive appropriate treatment for TB and HIV/AIDS. Upon recovery, they should also be relocated to work in areas with lowest risk of $\mathrm{TB}$ and in health settings where appropriate WHO-recommended infection control measures are being implemented.

The obvious limitations of our study, given its retrospective design and ascertainment bias, are the inability to calculate prevalence estimates, or to precisely delineate whether the XDR-TB in HCWs was acquired nosocomially or from the community. The physical, social and economic consequences of HCWs acquiring XDR-TB can be very severe and affect their daily lives.

Thus, it becomes important for the South African government to take immediate measures to provide the resources required to enable all hospitals and clinics providing TB care to follow and to implement the recommended WHO infection control procedures (WHO Report, 2009). This will go a long way in protecting HCWs and other inpatients from acquiring MDR-/XDR-TB from those patients and HCWs who have active disease. Furthermore, it is important that all health facility laboratories be equipped with the newer rapid diagnostic testing platforms now available, so that all patients and HCWs suspected of having TB need can be identified early, and appropriately isolated, to minimize the risk of transmission within hospitals and in the community.

\section{Conclusions}

In the Western and Eastern Cape provinces of South Africa $\mathrm{XDR}-\mathrm{TB}$ is diagnosed rather late, does not appear to be related to HIV status and carries a high mortality. This underscores the need for the government to implement current WHO policy on TB infection control in all health care facilities in South Africa. There is also the need for making available rapid molecular biological DST for all HCWs and inpatients to rapidly detect, isolate, and treat all patients with MDR-/XDR-TB. Now that we have shown that HCWs succumb to XDR-TB, further studies to establish the source and mode of infection in HCWs, and the risk to their patients and families, are urgently required.

\section{Acknowledgements}

Keertan Dheda was supported by the South African National Research Foundation, the South African Medical Research Council, an EU FP7 Grant and the EDCTP. Alimuddin Zumla receives support from the EU, EuropeAID, EDCTP, UK-MRC and the NIHR UCL-HNHSTrust CBRC. Charlotte Kvasnovsky and Martie Van der Walt were supported by the South African MRC and Center for Disease Control through a cooperative agreement to MRC. Charlotte Kvasnovsky was supported by a grant from Emory School of Medicine.

\section{References}

Centers for Disease Control and Prevention (2006) Emergence of Mycobacterium tuberculosis with extensive resistance to second-line drugs - worldwide, 2000-2004. MMWR. Morbidity and Mortality Weekly Report 55, 301-305.

Dheda K, Shean K, Zumla A et al. (2010) Early treatment outcomes of extensively drug-resistant tuberculosis in South Africa are poor regardless of HIV status. The Lancet 375, 1798-1807.

Galgalo T, Dalal S, Cain KP et al. (2008) Tuberculosis risk among staff of a large public hospital in Kenya. The International Journal of Tuberculosis and Lung Disease: The Official Journal of the International Union against Tuberculosis and Lung Disease 12, 949-954.

Gandhi NR, Moll A, Sturm AW et al. (2006) Extensively drugresistant tuberculosis as a cause of death in patients co-infected with tuberculosis and HIV in a rural area of South Africa. Lancet 368, 1575-1580.

Jo KW, Woo JH, Hong Y et al. (2008) Incidence of tuberculosis among health care workers at a private university hospital in South Korea. The International Journal of Tuberculosis and Lung Disease: The Official Journal of the International Union against Tuberculosis and Lung Disease 12, 436-440.

Joshi R, Reingold AL, Menzies D \& Pai M (2006) Tuberculosisamong health-care workers in low- and middleincomecountries: a systematic review. PLoS Medicine 3, e494.

McEvoy C, van Helden P, Warren RM \& van Pittius N (2009) Evidence for a rapid rate of molecular evolution at the hypervariable and immunogenic Mycobacterium tuberculosis PPE38 gene hypervariable region. BME Evolutionary Biology 9, 237. $1-21$.

Menzies D, Joshi R \& Pai M (2007) Risk of tuberculosis infection and disease associated with work in health care settings. The International Journal of Tuberculosis and Lung Disease: The Official Journal of the International Union against Tuberculosis and Lung Disease 11, 593-605.

Naidoo S \& Jinabhai CC (2006) TB in health care workers in KwaZulu-Natal, South Africa. Int J Tuberc Lung Dis 10, 676682. 


\section{J. Jarand et al. XDR-TB among health care workers in South Africa}

Pai M, Kalantri S \& Dheda K (2006) New tools and emerging technologies for the diagnosis of tuberculosis: part II. Active tuberculosis and drug resistance. Expert Review of Molecular Diagnostics 6, 423-432.

Pillay Y \& Malhati P (2008) Health-worker salaries and incomes in sub-Saharan Africa. The Lancet 371, 632634.

Shisana O, Hall EJ, Maluleke R, Chauveau J \& Schwabe C (2004) HIV/AIDS prevalence among South African health workers. South African Medical Journal 94, 846-850.

Sotgiu G, Ferrara G, Matteelli A et al. (2009) Epidemiology and clinical management of XDR-TB: a systematic review by TBNET. European Respiratory Journal 33, 871-881.

Verver S, Warren RM, Beyers N et al. (2005) Rate of reinfection tuberculosis after successful treatment is higher than rate of new tuberculosis. American Journal of Respiratory and Critical Care Medicine 171, 1430-1435.
WHO (2010) Multidrug and Extensively Drug-resistant TB (M/XD-TB). 2010 Global Report on Surveillance and Response. WHO, Geneva. WHO/HTM/TB/2010.3.

WHO Report (2009) WHO policy on TB infection control in health care facilities, congregate settings and households. WHO/HTM/TB/2009.419.

WHO Report. World Health Organization (2010) M/XDR-TB Surveillance and Control: 2010 Global Update. Geneva, Switzerland.

Wilkinson D \& Gilks CF (1998) Increasing frequency of staff in a South African district hospital: impact of the HIV epidemic on the supply side of health care. Transactions of the Royal Society of Tropical Medicine and Hygiene 92, 500-502.

World Health Organization (2006) The global shortage of health workers and its impact. WHO Fact sheet No. 302. Geneva. April 2006. http://www.who.int/mediacentre/factsheets/fs302/ en/print.html, accessed April 2009.

Corresponding Author Keertan Dheda, Department of Medicine, H floor (Old Main Building), Groote Schuur Hospital, Observatory, Cape Town 7925, South Africa. Tel.: +27 21 4066509; Fax: +27 21 4486815; E-mail: keertan.dheda@uct.ac.za 